\title{
Flavor asymmetry of the nucleon sea
}

\author{
J. C. Peng \\ aPhysics Division, Los Alamos National Laboratory, \\ Los Alamos, New Mexico, 87545 U.S.A.
}

Recent deep inelastic scattering and Drell-Yan experiments have revealed a surprisingly large asymmetry between the up and down sea quark distributions in the nucleon. The current status of the flavor asymmetry of the nucleon sea is reviewed. Implications of various theoretical models and possible future measurements are also discussed.

\section{INTRODUCTION}

One of the most active areas of research in nuclear and particle physics during the last several decades is the study of quark and gluon distributions in the nucleons and nuclei. Several major surprises were discovered in Deep-Inelastic Scattering (DIS) experiments which profoundly changed our views of the partonic substructure of hadrons. In the early 1980's, the famous 'EMC' effect found in muon DIS provided the first unambiguous evidence that the quark distributions in nuclei are significantly different from those in free nucleons [1,2]. More recently, surprising results on the spin and flavor structures of the nucleons were discovered in DIS experiments. The so-called "spin crisis", revealed by the disagreement between the prediction of the Ellis-Jaffe sum rule and the polarized DIS experiments, has led to extensive theoretical and experimental efforts to understand the partonic content of proton's spin [3]. Subsequently, the observation [0] of the violation of the Gottfried sum rule [5] in DIS revealed a surprisingly large asymmetry between the up and down antiquark distributions in the nucleon, shedding new light on the origins of the nucleon sea.

In this article, we review the status of our current knowledge on the flavor dependence of the sea quark distributions in hadrons. In Section 2, we review the early studies of the nucleon sea in DIS and lepton-pair production. The crucial recent experiments establishing the up/down flavor asymmetry of the nucleon sea are also discussed in Section 2. Various theoretical models for explaining the $\bar{d} / \bar{u}$ asymmetry are described in Section 3. The implications of these models on other aspects of the parton structure functions are discussed in Section 4. Finally, we present future prospects in Section 5, followed by conclusion in Section 6.

\section{EXPERIMENTAL EVIDENCE FOR $\bar{d} / \bar{u}$ ASYMMETRY}

The earliest parton models assumed that the proton sea was flavor symmetric, even though the valence quark distributions are clearly flavor asymmetric. Inherent in this 
assumption is that the content of the sea is independent of the valence quark's composition. Therefore, the proton and neutron were expected to have identical sea-quark distributions. The flavor symmetry assumption was not based on any known physics, and it remained to be tested by experiments. Neutrino-induced charm production experiments [6,7], which are sensitive to the $s \rightarrow c$ process, provided strong evidences that the strange-quark content of the nucleon is only about half of the up or down sea quarks. Such flavor asymmetry is attributed to the much heavier strange-quark mass compared to the up and down quarks. The similar masses for the up and down quarks suggest that the nucleon sea should be nearly up-down symmetric.

The issue of the equality of $\bar{u}$ and $\bar{d}$ was first encountered in measurements of the Gottfried integral [5], defined as

$$
I_{G}=\int_{0}^{1}\left[F_{2}^{p}\left(x, Q^{2}\right)-F_{2}^{n}\left(x, Q^{2}\right)\right] / x d x
$$

where $F_{2}^{p}$ and $F_{2}^{n}$ are the proton and neutron structure functions measured in DIS experiments. Assuming charge symmetry, $I_{G}$ can be expressed in terms of the valence and sea quark distributions of the proton as:

$$
I_{G}=\frac{1}{3} \int_{0}^{1}\left[u_{v}\left(x, Q^{2}\right)-d_{v}\left(x, Q^{2}\right)\right] d x+\frac{2}{3} \int_{0}^{1}\left[\bar{u}\left(x, Q^{2}\right)-\bar{d}\left(x, Q^{2}\right)\right] d x .
$$

Under the assumption of a $\bar{u}, \bar{d}$ flavor-symmetric sea in the nucleon, the Gottfried Sum Rule (GSR) [5], $I_{G}=1 / 3$, is obtained.

The first measurements of the Gottfried integral were carried out in the 1970s [8,9] soon after the discovery of scaling in DIS. Although the large systematic errors associated with the unmeasured small- $x$ region prevented a sensitive test of the GSR, Field and Feynman [10 nevertheless interpreted the early SLAC data as strong indications that GSR is violated and that the $\bar{u}$ and $\bar{d}$ distributions in the proton are different. The SLAC DIS experiments were followed by several muon-induced DIS experiments at Fermilab and at CERN. Despite the fact that all measurements of Gottfried integral consistently showed a value lower than $1 / 3$, the large systematic errors prevented a definitive conclusion. As a result, all parametrizations [11 13 of the parton distributions based on global fits to existing data before 1990 assumed a symmetric $\bar{u}, \bar{d}$ sea.

The most accurate test of the GSR was reported in 1991 by the New Muon Collaboration (NMC) [1], which measured $F_{2}^{p}$ and $F_{2}^{n}$ over the region $0.004 \leq x \leq 0.8$. They determined the Gottfried integral to be $0.235 \pm 0.026$, significantly below $1 / 3$. This surprising result has generated much interest.

Although the violation of the GSR can be explained by assuming unusual behavior of the parton distributions at very small $x$, a more natural explanation is to abandon the assumption $\bar{u}=\bar{d}$. Specifically, the NMC result implies

$$
\int_{0}^{1}[\bar{d}(x)-\bar{u}(x)] d x=0.148 \pm 0.039
$$

It should be emphasized that only the integral of $\bar{d}-\bar{u}$ was deduced from the DIS measurements. The $x$ dependence of $\bar{d}-\bar{u}$ remained unspecified. 


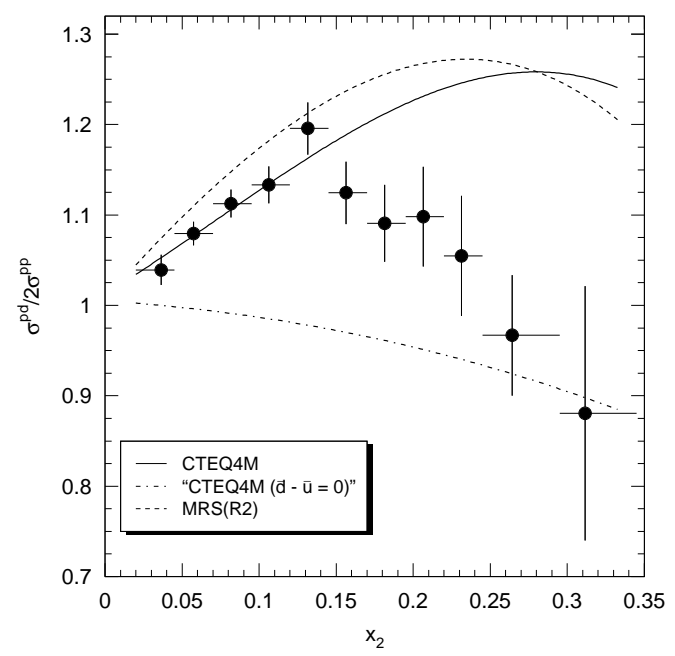

Figure 1. The ratio $\sigma^{p d} / 2 \sigma^{p p}$ of Drell-Yan cross sections vs. $x_{2}$ from Fermilab E866 [18]. The curves are next-to-leading order calculations, weighted by acceptance, of the DrellYan cross section ratio using the CTEQ4M [20] and MRS(R2) [21] parton distributions. In the lower CTEQ4M curve, $\bar{d}-\bar{u}$ was set to 0 to simulate a symmetric sea.

The proton-induced Drell-Yan (DY) process provides an independent means to probe the flavor asymmetry of the nucleon sea [14]. An important advantage of the DY process is that the $x$ dependence of $\bar{d} / \bar{u}$ can be determined. It is interesting to note that, as early as 1981, Fermilab E288 [15] reported evidence for a $\bar{d} / \bar{u}$ asymmetry, based on a measurement of the $p+d$ DY cross section. However, this interpretation depended sensitively on assumptions about the shape of the valence quark distributions and was not conclusive. Later, the Fermilab E772 collaboration [16] compared the DY yields from isoscalar targets with that from a neutron-rich (tungsten) target, and constraints on the nonequality of $\bar{u}$ and $\bar{d}$ in the range $0.04 \leq x \leq 0.27$ were set. More recently, the CERN experiment NA51 [17] carried out a comparison of the DY muon pair yield from hydrogen and deuterium using a $450-\mathrm{GeV} / \mathrm{c}$ proton beam. They found that $\bar{u} / \bar{d}=0.51 \pm 0.04 \pm 0.05$ at $\langle x\rangle=0.18$, a surprisingly large difference between the $\bar{u}$ and $\bar{d}$.

A DY experiment (E866), aiming at higher statistical accuracy and wider kinematic coverage than NA51, was recently completed 18,19 at Fermilab. This experiment also measured the DY muon pairs from $800-\mathrm{GeV} / \mathrm{c}$ protons interacting with liquid deuterium and hydrogen targets. The acceptance of the spectrometer was largest for $x_{F}=x_{1}-$ $x_{2}>0$. In this kinematic regime the DY cross section is dominated by the annihilation of a beam quark with a target antiquark. The DY cross section ratio at large $x_{F}$ is approximately given as

$$
\frac{\sigma_{D Y}(p+d)}{2 \sigma_{D Y}(p+p)} \approx \frac{1}{2}\left(1+\frac{\bar{d}\left(x_{2}\right)}{\bar{u}\left(x_{2}\right)}\right) .
$$

The ratio is unity when $\bar{d}=\bar{u}$. Figure 1 shows that the E866 measurement of this ratio clearly exceeds unity for an appreciable range in $x_{2}$.

Using an iterative procedure [19], values for $\bar{d} / \bar{u}$ were extracted by the E866 collaboration at $Q^{2}=54 \mathrm{GeV}^{2} / \mathrm{c}^{2}$. These are shown in Figure 2 along with the NA51 measurement. For $x<0.15, \bar{d} / \bar{u}$ increases linearly with $x$ and is in good agreement with 


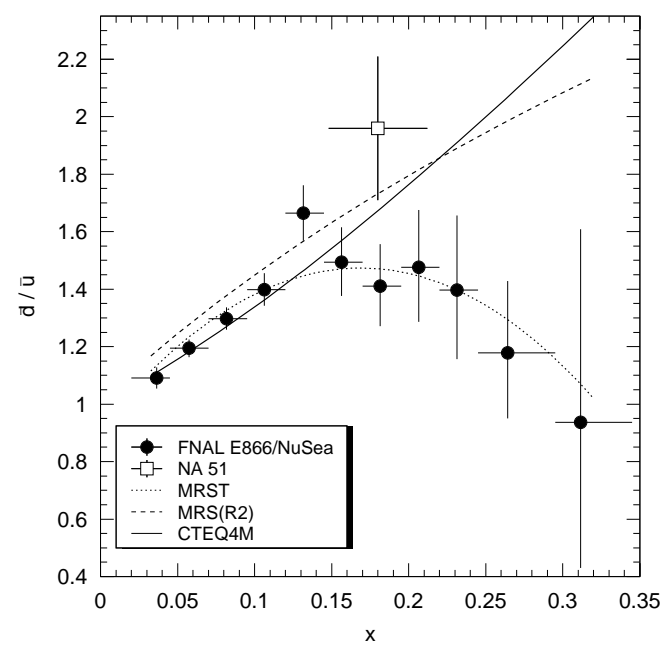

Figure 2. The ratio of $\bar{d} / \bar{u}$ in the proton as a function of $x$ extracted from the Fermilab E866 [18 cross section ratio. The curves are from various parton distributions. Also shown is the result from NA51 [17], plotted as an open box.

the CTEQ4M 20 and MRS(R2) 21] parameterizations. However, a distinct feature of the data, not seen in either parameterization, is the rapid decrease toward unity of $\bar{d} / \bar{u}$ beyond $x=0.2$.

The $\bar{d} / \bar{u}$ ratio, along with the CTEQ4M values for $\bar{d}+\bar{u}$, was used to obtain $\bar{d}-\bar{u}$ over the region $0.02<x<0.345$ (Figure 3). From the results shown in Figure 3, one can obtain an independent determination [19] of the integral of Equation 3. E866 finds $0.100 \pm 0.007 \pm 0.017$, consistent with, but roughly $2 / 3$ of the value deduced by NMC.

The HERMES collaboration recently reported a semi-inclusive DIS measurement of charged pions from hydrogen and deuterium targets [22]. Based on the differences between charged-pion yields from the two targets, the ratio $(\bar{d}-\bar{u}) /(u-d)$ is determined in the kinematic range, $0.02<x<0.3$ and $1 \mathrm{GeV}^{2} / \mathrm{c}^{2}<Q^{2}<10 \mathrm{GeV}^{2} / \mathrm{c}^{2}$. The HERMES results for $\bar{d}-\bar{u}$, shown in Figure 3, are consistent with the E866 results obtained at significantly higher $Q^{2}$.

\section{ORIGINS OF THE $\bar{d} / \bar{u}$ ASYMMETRY}

The earliest experiments indicated that the value of the Gottfried integral might be less than $1 / 3$, leading to speculation regarding the origin of this reduction. Field and Feynman suggested [10] that it could be due to Pauli blocking in so far as $u \bar{u}$ pairs would be suppressed relative to $d \bar{d}$ pairs because of the presence of two $u$-quarks in proton as compared to a single $d$-quark. Ross and Sachrajda [23] questioned that this effect would be appreciable because of the large phase-space available to the created $q \bar{q}$ pairs. They also showed that perturbative QCD would not produce a $\bar{d} / \bar{u}$ asymmetry. Steffens and Thomas [24] recently looked into this issue, explicitly examining the consequences of Pauli blocking. They similarly concluded that the blocking effects were small.

A natural origin for this flavor asymmetry is the virtual states of the proton containing isovector mesons. This point appears to have first been made by Thomas in a publication [25] treating $\mathrm{SU}(3)$ symmetry breaking in the nucleon sea. Sullivan [26] previously showed 


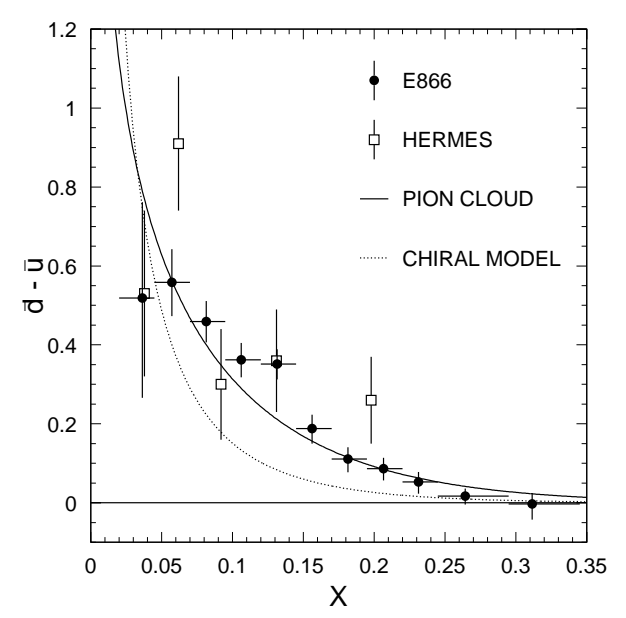

Figure 3. Comparison of the E866 18 $\bar{d}-\bar{u}$ results at $Q^{2}=54 \mathrm{GeV}^{2} / \mathrm{c}^{2}$ with the predictions of pion-cloud and chiral models as described in the text. The data from HERMES 22] are also shown.

that in DIS virtual mesons scale in the Bjorken limit and contribute to the nucleon structure function. Following the publication of the NMC result, many papers treated virtual mesons as the origin of the $\bar{d} / \bar{u}$ asymmetry (see [27,28] for recent reviews). Here the $\pi^{+}(\bar{d} u)$ cloud, dominant in the process $p \rightarrow \pi^{+} n$, leads to an excess of $\bar{d}$ sea.

A different approach for including the effects of virtual mesons has been presented by Eichten et al. [29] and further investigated by other authors [30,31. In chiral perturbation theory, the relevant degrees of freedom are constituent quarks, gluons, and Goldstone bosons. In this model, a portion of the sea comes from the couplings of Goldstone bosons to the constituent quarks, such as $u \rightarrow d \pi^{+}$and $d \rightarrow u \pi^{-}$. The excess of $\bar{d}$ over $\bar{u}$ is then simply due to the additional valence $u$ quark in the proton.

The $x$ dependences of $\bar{d}-\bar{u}$ and $\bar{d} / \bar{u}$ obtained by E866 provide important constraints for theoretical models. Figure 3 compares $\bar{d}(x)-\bar{u}(x)$ from E866 with a virtual-pion model calculation, following the procedure detailed by Kumano [32]. Figure 3 (dotted curve) also shows the predicted $\bar{d}-\bar{u}$ from the chiral model. We follow the formulation of Szczurek et al 31] to calculate $\bar{d}(x)-\bar{u}(x)$ at $Q^{2}=0.25 \mathrm{GeV}^{2} / \mathrm{c}^{2}$ and then evolve the results to $Q^{2}=54 \mathrm{GeV}^{2} / \mathrm{c}^{2}$. The chiral model places more strength at low $x$ than does the virtual-pion model. This difference reflects the fact that the pions are softer in the chiral model, since they are coupled to constituent quarks, that carry only a fraction of the nucleon momentum. The $x$ dependence of the E866 data favors the virtual-pion model over the chiral model, suggesting that correlations between the chiral constituents should be taken into account.

Recently, the flavor asymmetry of the nucelon sea was computed in the large- $N_{c}$ limit, where the nucleon is described as a soliton of an effective chiral theory [33, 34. In this chiral quark-soliton model, the flavor non-singlet distribution, $\bar{d}(x)-\bar{u}(x)$, appears in the next-to-leading order of the $1 / N_{c}$ expansion [35,36]. The E866 $\bar{d}(x)-\bar{u}(x)$ data were shown to be well described by this model [34.

Instantons have been known as theoretical constructs since the seventies 37 39. The collision between a quark and an instanton flips the helicity of the quark while creating 
a $q \bar{q}$ pair of different flavor. Thus, interaction between a $u$ quark and an instanton results in a $u$ quark of opposite helicity and either a $d \bar{d}$ or $s \bar{s}$ pair. Such a model has the possibility of accounting for both the flavor asymmetry and the "spin crisis" 40. However, the prediction 41] at large $x, \bar{d}(x) / \bar{u}(x) \rightarrow 4$, is grossly violated by experiment (see Figure 2). Thus, it appears that while instantons have the possibility for accounting for flavor and spin anomalies, the approach is not yet sufficiently developed for a direct comparison.

\section{IMPLICATIONS OF THE MESON-CLOUD MODELS}

Models in which virtual mesons are admitted as degrees of freedom have implications that extend beyond the $\bar{d} / \bar{u}$ flavor asymmetry addressed above. They create hidden strangeness in the nucleon via such virtual processes as $p \rightarrow \Lambda+K^{+}, \Sigma+K$, etc. Such processes are of considerable interest as they imply different $s$ and $\bar{s}$ parton distributions in the nucleon, a feature not found in gluonic production of $s \bar{s}$ pairs. This subject has received a fair amount of attention in the literature 42 46 but experiments have yet to clearly identify such a difference.

A difference between the $s$ and $\bar{s}$ distribution can be made manifest by direct measurement of the $s$ and $\bar{s}$ parton distribution functions in DIS neutrino scattering, or in the measurement of the $q^{2}$ dependence of the strange quark contribution $\left(F_{1 s}^{p}\left(q^{2}\right)\right)$ to the proton charge form factor. This latter case follows from a suggestion of Kaplan and Manohar 447 regarding the new information contained in the weak neutral current form factors of the nucleon. Measurement of these form factors allows extraction of the strangeness contribution to the nucleon's charge and magnetic moment and axial form factors. The portion of the charge form factor $F_{1 s}^{p}\left(q^{2}\right)$ due to strangeness clearly is zero at $q^{2}=0$, but if the $s$ and $\bar{s}$ distributions are different the form factor becomes non-zero at finite $q^{2}$. These "strange" form factors can be measured in neutrino elastic scattering 448 from the nucleon, or by selecting the parity-violating component of electron-nucleon elastic scattering, as is now being done at the Bates [49] and Jefferson Laboratories [50].

It is worth pointing out that there is a relationship between the parton distributions and the form factors of a hadron. If the neutron's charge form factor is explained in terms of a particular meson-baryon expansion, then one should expect that the expansion is consistant with the neutron's partonic structure.

The chiral and the meson-cloud models both predict that the $\bar{u}$ and $\bar{d}$ quarks will carry negligible amount of the proton's spin [19,29,51]. In striking contrast, the chiralquark model predicts [54] a significantly polarized sea with a large flavor asymmetry. In particular, this model predicts a larger values for $\Delta \bar{u}-\Delta \bar{d}$ than for $\bar{d}-\bar{u}$. Both the SMC [52] and the HERMES [53] experiments attempted to extract the sea-quark polarizations via semi-inclusive polarized DIS measurements, and the results indicate small sea-quark polarization consistent with zero. However, as pointed out in Ref. [54, large uncertainties are associated with certain assumptions made in the extraction. Future DY and $W^{ \pm}$production experiments at RHIC could clearly test these models [55].

The observation of a large $\bar{d} / \bar{u}$ asymmetry in the proton has motivated Alberg et al. 56,57 to consider the sea-quark distributions in the $\Sigma$. The meson-cloud model implies a $\bar{d} / \bar{u}$ asymmetry in the $\Sigma^{+}$even larger than that of the proton. However, the 
opposite effect is expected from SU(3) symmetry. Although relatively intense $\Sigma^{+}$beams have been produced for recent experiments at Fermilab, this experiment appears to be very challenging because of large pion, kaon, and proton contaminations in the beam.

\section{FUTURE PROSPECT}

The interplay between the perturbative and non-perturbative components of the nucleon sea remains to be better determined. Since the perturbative process gives a symmetric $\bar{d} / \bar{u}$ while a non-perturbative process is needed to generate an asymmetric $\bar{d} / \bar{u}$ sea, the relative importance of these two components is directly reflected in the $\bar{d} / \bar{u}$ ratios. Thus, it would be very important to extend the DY measurements to kinematic regimes beyond the current limits.

The new $120 \mathrm{GeV}$ Fermilab Main Injector (FMI) and the proposed $50 \mathrm{GeV}$ Japanese Hadron Facility [58] (JHF) present opportunities for extending the $\bar{d} / \bar{u}$ measurement to larger $x(x>0.25)$. For given values of $x_{1}$ and $x_{2}$ the DY cross section is proportional to $1 / s$, hence the DY cross section at $50 \mathrm{GeV}$ is roughly 16 times greater than that at $800 \mathrm{GeV}$ ! The values of $\bar{d} / \bar{u}$ over the region $0.25<x<0.7$ could indeed be obtained at FMI and JHF [59,60, and these data would be extremely valuable for testing various theoretical models.

At the other end of the energy scale, RHIC will operate soon in the range $50 \leq \sqrt{s} \leq 500$ $\mathrm{GeV} /$ nucleon. The capability of accelerating and colliding a variety of beams from $p+p$, $p+A$, to $A+A$ at RHIC offers a unique opportunity to extend the DY $\bar{d} / \bar{u}$ measurement to very small $x$. Furthermore, an interesting quantity to be measured at RHIC is the ratio of the $p+p \rightarrow W^{+}+X$ and $p+p \rightarrow W^{-}+X$ cross sections [61]. It can be shown that this ratio is very sensitive to $\bar{d} / \bar{u}$. An important feature of the $W$ production asymmetry in $p+p$ collision is that it is completely free from the assumption of charge symmetry.

As discussed earlier, an interesting consequence of the meson-cloud model is that the $s$ and $\bar{s}$ distributions in the proton could have very different shapes, even though the net amount of strangeness in the proton vanishes. By comparing the $\nu$ and $\bar{\nu}$ induced charm production, the CCFR collaboration found no difference between the $s$ and $\bar{s}$ distributions [62]. More precise future measurements would be very helpful. Dimuon production experiments using $K^{ \pm}$beams might provide an independent determination of the $s / \bar{s}$ ratio of the proton, provided that our current knowledge on valence-quark distributions in kaons is improved. Ongoing measurements of $F_{1 s}^{p}$ via parity-violating electron-nucleon scattering should shed much light on the possible difference between $s$ and $\bar{s}$ distributions.

DY and $W^{ \pm}$production in polarized $p+p$ collision are planned at RHIC 63 and they have great potential for providing qualitatively new information about antiquark polarization. At large $x_{F}$ region $\left(x_{F}>0.2\right)$, the longitudinal spin asymmetry $A_{L L}$ in the $\vec{p}+\vec{p} \mathrm{DY}$ process is given by [64,65]

$$
A_{L L}^{D Y}\left(x_{1}, x_{2}\right) \approx g_{1}\left(x_{1}\right) / F_{1}\left(x_{1}\right) \times \frac{\Delta \bar{u}}{\bar{u}}\left(x_{2}\right),
$$

where $g_{1}(x)$ is the proton polarized structure function measured in DIS, and $\Delta \bar{u}(x)$ is the polarized $\bar{u}$ distribution function. Eq. using polarized DY at RHIC. 


\section{CONCLUSION}

The flavor asymmetry of the nucleon sea has been clearly established by recent DIS and DY experiments. The surprisingly large asymmetry between $\bar{u}$ and $\bar{d}$ is unexplained by perturbative QCD. Thus far, three distinct explanations have been offered in the literature for the origin of the observed $\bar{d}, \bar{u}$ asymmetry. The first is Pauli blocking which, while appealing, is difficult to calculate and appears to produce too small an effect to be the sole origin of the large observed asymmetry. The second involves virtual isovector mesons, mostly pions, in the nucleon. Such descriptions necessarily require non-perturbative QCD and, apart from lattice gauge calculations, demand additional parameters and possess systematic uncertainties. However, as these virtual mesons readily generate a large $\bar{d} / \bar{u}$ asymmetry, many authors, using a variety of techniques to invoke and justify their approachs, have obtained qualitative agreement with the experimental measurements. The third explanation involves instantons, but the theory is not sufficiently developed to allow quantitative comparison to the asymmetry data. Future experiments will test and refine these models. They will further illuminate the interplay between the perturbative and non-perturbative nature of the nucleon sea.

\section{REFERENCES}

1. J.J. Aubert et al., Phys. Lett. B123 (1983) 295.

2. D.F. Geesaman, K. Saito, A.W. Thomas, Annu. Rev. Nucl. Part. Sci. 45 (1995) 337.

3. E. Hughes and R. Voss, Annu. Rev. Nucl. Part. Sci. 49 (1999) 303.

4. P. Amaudruz et al., Phys. Rev. Lett. 66 (1991) 2712; M. Arneodo et al., Phys. Rev. D55 (1994) R1.

5. K. Gottfried, Phys. Rev. Lett. 18 (1967) 1174.

6. H. Abromowicz et al., Z. Phys. C15 (1982) 19.

7. J.M. Conrad, M.H. Shaevitz, T. Bolton, Rev. Mod. Phys. 70 (1998) 1341.

8. E.D. Bloom et al., Proc. 15th Int. Conf. on High Energy Phys., Kiev, USSR (1970).

9. E.D. Bloom, Proc. 6th Int. Sym. on Electron and Photon Interactions at High Energies, edited by H. Rollnik and W. Pfeil (North-Hollan, Amsterdam, 1974) 227.

10. R.D. Field and R.P. Feynman, Phys. Rev. D15 (1977) 2590.

11. D.W. Duke and J.F. Owens, Phys. Rev. D30 (1984) 49.

12. E. Eichten, I. Hinchliffe, K. Lane, C. Quigg, Rev. Mod. Phys. 56 (1984) 579.

13. P. Aurenche et al., Phys. Rev. D39 (1989) 3275.

14. S.D. Ellis and W.J. Stirling, Phys. Lett. B256 (1991) 258.

15. A.S. Ito et al., Phys. Rev. D23 (1981) 604.

16. P.L. McGaughey et al., Phys. Rev. Lett. 69 (1991) 1726.

17. A. Baldit et al., Phys. Lett. B332 (1994) 244.

18. E.H. Hawker et al., Phys. Rev. Lett. 80 (1998) 3715.

19. J.C. Peng et al., Phys. Rev. D58 (1998) 092004.

20. H.L. Lai et al., Phys. Rev. D55 (1997) 1280.

21. A.D. Martin, R.G. Roberts, W.J. Stirling, Phys. Lett. B387 (1996) 419.

22. K. Ackerstaff K et al., Phys. Rev. Lett. 81 (1998) 5519.

23. D.A. Ross and C.T. Sachrajda, Nucl. Phys. B149 (1979) 497.

24. F.M. Steffens and A.W. Thomas, Phys. Rev. C55 (1997) 900. 
25. A.W. Thomas, Phys. Lett. B126 (1983) 97.

26. J.D. Sullivan, Phys. Rev. D5 (1972) 1732.

27. S. Kumano, Phys. Rept. 303 (1998) 183.

28. J.C. Peng and G.T. Garvey, hep-ph/9912370 (1999).

29. E.J. Eichten, I. Hinchliffe, C. Quigg, Phys. Rev. D 45 (1992) 2269; 47 (1993) R747.

30. T.P. Cheng and L.F. Li, Phys. Rev. Lett. 74 (1995) 2872.

31. A. Szczurek, A. Buchmans, A. Faessler, J. Phys. C22 (1996) 1741.

32. S. Kumano, Phys. Rev. D43 (1991) 3067; 43 (1991) 59; S. Kumano, J.T. Londergan, Phys. Rev. D44 (1991) 717.

33. M. Wakamatsu and T. Kubota, Phys. Rev. D57 (1998) 5755.

34. P.V. Pobylitsa et al., Phys. Rev. D59 (1999) 034024.

35. D.I. Diakonov et al., Nucl. Phys. B480 (1996) 341.

36. D.I. Diakonov et al., Phys. Rev. D56 (1997) 4069.

37. A.A. Belavin et al., Phys. Lett. B59 (1975) 85.

38. G. t'Hooft, Phys. Rev. Lett .37 (1976) 8.

39. T. Schaefer and E. Shuryak, Rev. Mod. Phys. 70 (1998) 323.

40. S. Forte, Phys. Lett. B224 (1989) 189.

41. A.E. Dorokhov and N.I. Kochelev, Phys. Lett. B259 (1991) 335; B335 (1993) 167.

42. H. Holtzman, A. Szczurek and J. Speth, Nucl. Phys. A596 (1996) 631.

43. A.I. Signal and A.W. Thomas, Phys. Lett. B191 (1987) 205.

44. M. Burkardt and B.J. Warr, Phys. Rev. D45 (1992) 958.

45. X.D. Ji and J. Tang, Phys. Lett. B362 (1995) 182.

46. S. Brodsky and B-Q. Ma, Phys. Lett. B381 (1996) 317.

47. D. Kaplan and A. Manohar, Nucl. Phys. B310 (1988) 527.

48. G. Garvey, W. Louis, H. White, Phys. Rev. C48 (1993) 761.

49. B. Mueller et al., Phys. Rev. Lett. 78 (1997) 3824.

50. K. Aniol et al., Phys. Rev. Lett. 82 (1999) 1096.

51. T.P. Cheng and L.F. Li, Phys. Lett. B366 (1996) 365.

52. B. Adeva et al., Phys. Lett. 420 (1998) 180.

53. K. Akerstaff et al., hep-ex/9906035 (1999).

54. B. Dressler et al., hep-ph/9909541 (1999).

55. B. Dressler et al., hep-ph/9910464 (1999).

56. M. Alberg et al., Phys. Lett. B389 (1996) 367.

57. M. Alberg, T. Falter, E.M. Henley, Nucl. Phys. A644 (1998) 93.

58. JHF Project Office. Proposal for Japan Hadron Facility, KEK Rep. 97-3 (1997).

59. D. Geesaman et al., Fermilab proposal P906 (1999).

60. J.C. Peng, G.T. Garvey, J.M. Moss, S. Sawada, J. Chiba, hep-ph/0007341 (2000).

61. J.C. Peng and D.M. Jansen, Phys. Lett. B 354 (1995) 460.

62. A.O. Bazarko et al., Z. Phys. C65 (1995) 189.

63. G. Bunce et al. Part. World 3 (1992) 1.

64. J.M. Moss, Int. Conf. Polarization Phenomena in Nucl. Phys., AIP Conf. Proc. 339 (1994) 721.

65. P.L. McGaughey, J.M. Moss and J.C. Peng, Annu. Rev. Nucl. Part. Sci. 49 (1999) 217. 